\title{
Interval Arithmetic and Computational Science: Performance Considerations
}

\author{
Alistair P. Rendell, Bill Clarke, and Josh Milthorpe \\ Department of Computer Science, Australian National University \\ Canberra ACT0200, Australia \\ alistair.rendell@anu.edu.au
}

\begin{abstract}
Interval analysis is an alternative to conventional floating-point computations that offers guaranteed error bounds. Despite this advantage, interval methods have not gained widespread use in large scale computational science applications. This paper addresses this issue from a performance perspective, comparing the performance of floating point and interval operations for some small computational kernels. Particularly attention is given to the Sun Fortran interval implementation, although the strategies introduced here to enhance performance are applicable to other interval implementations. Fundamental differences in the operation counts and memory references requirements of interval and floating point codes are discussed.
\end{abstract}

\section{Introduction}

The majority of science is concerned with physical phenomena, such as velocity, temperature, or pressure that are by their very nature continuous. Meanwhile computations of these quantities are performed using the discrete environment of the digital computer. To bridge this divide it is normal to approximate values to a specified precision using a finite set of machine-representable numbers. Inherent in this process is the concept of a rounding error, the effect of which can be hard to predict a priori.

Currently most scientific codes use IEEE 754 double precision arithmetic [1] and give relatively little or no attention to the effects of rounding errors. While this may have been okay in the past, on today's machines that are capable of multi teraflop ( $>10^{12}$ operations) per second and with double precision arithmetic providing just 15 16 significant figures, it is easy to see the potential for rounding errors to compound and become as large as the computed quantities themselves.

Interval arithmetic is a fundamentally different approach to floating point computations that that was first proposed by R.E. Moore in 1965 [2], but is yet to achieve widespread use in computational science applications. The idea is to represent a floating point number by two floating point numbers corresponding to a lower and upper bound (referred to as the infima and suprema respectively). In concept it is identical to expressing the uncertainty in a quantity as $1.234 \pm 0.001$, except that the interval representation would be written as $[1.233,1.235]$ since this is easier to manipulate. The basic rules for interval addition and multiplication are as follows: 


$$
\begin{aligned}
{[\underline{x}, \bar{x}]+[\underline{y}, \bar{y}]=} & {[\underline{x}+\underline{y}, \bar{x}+\bar{y}] } \\
{[\underline{x}, \bar{x}] \times[\underline{y}, \bar{y}]=} & {[\min (\underline{x} \times \underline{y}, \underline{x} \times \bar{y}, \bar{x} \times \underline{y}, \bar{x} \times \bar{y}),} \\
& \max (\underline{x} \times \underline{y}, \underline{x} \times \bar{y}, \bar{x} \times \underline{y}, \bar{x} \times \bar{y})]
\end{aligned}
$$

Division is slightly more complicated, particularly if the interval appearing in the denominator spans 0 , and for details the reader is referred to [3]. Suffice it to say that anything that can be computed using floating point arithmetic can also be computed using intervals, although there are some fundamental differences between interval and standard floating point arithmetic. For instance interval arithmetic is not distributive [3], so the order in which interval operations are performed can lead to different interval results; the trick is to find the order of operations that give rise to the narrowest or sharpest possible interval result.

Interval computations can be used to bound errors from all sources, including input uncertainty, truncation and rounding errors. Given this apparent advantage it is perhaps somewhat surprising that intervals are not already widely use in computational science. The reason for this is arguably twofold; first difficulties associated with designing interval algorithms that produce narrow or sharp interval results, and second the performance penalty associated with use of intervals. In this paper we do not address the former, but instead focus on the performance penalty associated with performing interval operations on a computer.

There is a range of software products that are designed to support interval computations on popular architectures (see reference 4). Most of these (e.g. FILIB [5]) take the form of $\mathrm{C}++$ template libraries and as such have obvious limitations for widespread use in computational science. A much more attractive alternative is provided by the Sun Studio Fortran compiler [6], as this has been extended to provide support for an interval data type. Technically this means that using this compiler it is possible to make an interval version of an existing computational science application code by simply changing all floating point data types to intervals. While we are investigating such things [7], in this paper the goal is to determine the likely performance penalty a typical user might see if he/she where to make such a switch.

\section{Simple Interval Operations}

Our first objective was to compare asymptotic performance for pipelined floating point and interval operations. This was done using the following simple Fortran loop:

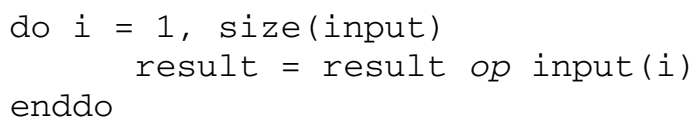

where result and input were either double precision floats or double precision intervals, and op was either addition or multiplication. This loop was placed in a separate procedure that was called many times, with care taken when initializing vector input to ensure that result did not over or underflow. 
The benchmark code was compiled using version 8.1 of the Sun Fortran compiler (part of Sun Studio 10) with options:

$$
\text { -fast }- \text { xarch }=v 9 b \quad[-x i a]
$$

where fast is a macro corresponding to a number of different optimization flags, $\mathrm{V} 9 \mathrm{~b}$ requests a 64-bit binary, and xia indicates that the compiler should use interval support. Timing results for this simple benchmark run on a $900 \mathrm{MHz}$ UltraSPARC III $\mathrm{Cu}$ system are given in Table 1. As intervals require twice the storage of the equivalent floating point values results obtained using a given floating point vector length are compared with interval results obtained using half that vector length. Results for three different vector sizes are considered. For the small case the data resides in the $64 \mathrm{~KB}$ level 1 cache, for the medium case the data resides in the $8 \mathrm{MB}$ level 2 cache, while for the large case data will be drawn from main memory.

Table 1. Performance (nsec) for summation and product benchmarks compiled using Sun Fortran 958.1 and run on a 900MHz UltraSPARC III Cu under Solaris 5.10

\begin{tabular}{rcccccc}
\hline & \multicolumn{3}{c}{ Floating Point } & & \multicolumn{3}{c}{ Interval } \\
\cline { 3 - 4 } Name & Input Size & Sum & Product & Input Size & Sum & Product \\
\hline Small & 2000 & 1.7 & 1.7 & 1000 & 35.7 & 102 \\
Medium & 512000 & 1.7 & 1.7 & 256000 & 57.4 & 122 \\
Large & 4096000 & 7.2 & 7.0 & 2048000 & 58.6 & 124 \\
\hline
\end{tabular}

The timing results for the floating point benchmarks are identical for both the small and medium vector size, reflecting the fact that the compiler has used prefetch instructions (evident in the assembly) to mask the cost of retrieving data from level 2 cache. For the large data set the performance drops markedly due to level 2 cache misses (confirmed using hardware performance counters). For the interval benchmark while there appears to be some cache effects, the most notably observation is the huge performance penalty associated with using intervals compared to floating point variables.

This prompts the obvious question, what should be the relative cost of performing an interval operation over the equivalent floating point operation? From equation 1 it would appear that an interval addition should cost at least twice that of a floating point addition - since two data items must be loaded into registers and two floating point additions performed (one on the infimas and one on the supremas). Some extra cost might also be expected since when adding the infimas the result must be rounded down, while when adding the supremas the result must be rounded up. For the interval product the expected performance is a little more difficult to predict; Equation 2 suggests the need to form 8 possible products and then a number of comparisons ino order to obtain the minimum and maximum of these. While we will return to this issue in more detail it does appear that a slowdown of 21 for the small interval addition benchmark and 60 for the small interval product benchmark over their equivalent floating point versions is excessive. 
Indeed it is already clear from these simple results that for interval arithmetic to be widely used for large scale computational science applications the performance penalty associated with use of intervals needs to be substantially reduced. From above and via close inspection of the generated assembly there appears to be four main reasons why the Sun interval code is slower than its equivalent floating point code:

1. The inherent cost of interval arithmetic over equivalent floating point (at least 2 for addition and 8 for multiplication as discussed above);

2. The fact that the Sun compiler translates each interval operation into a function call;

3. A lack of loop unrolling due in part to the use of procedures to implement the basic interval operations; and

4. Need for additional instructions to change the rounding mode every time an interval operation is performed.

To quantify these effects Table 2 gives timing results for the floating point benchmark modified to run in an analogous fashion to the interval code. That is the compiler settings are first adjusted to prevent loop unrolling, then the benchmark is re-written so that each floating point addition or multiplication is performed in a separate function, and then additional calls are inserted into this separate function to switch rounding mode every time it is called. These results coupled with the larger inherent cost of interval operations explain the bulk of the performance differences between the floating point and interval benchmarks (i.e. the differences seen in Table 1).

Table 2. Performance (nsec) of modified sum and product floating-point benchmarks compiled using Sun Fortran 958.1 and run on a 900MHz UltraSPARC III Cu under Solaris 5.10. See text for details of modifications.

\begin{tabular}{rcccccccc}
\hline & \multicolumn{2}{c}{ Initial } & \multicolumn{2}{c}{ No Unrolling } & \multicolumn{2}{c}{+ Function Call } & \multicolumn{2}{c}{ +Rounding } \\
Size & Sum & Prod & Sum & Prod & Sum & Prod & Sum & Prod \\
\hline Small & 1.7 & 1.7 & 4.5 & 5.6 & 24.5 & 24.5 & 28.9 & 26.9 \\
Medium & 1.7 & 1.7 & 4.5 & 5.6 & 29.4 & 30.7 & 33.8 & 32.4 \\
Large & 7.2 & 7.0 & 7.0 & 7.8 & 54.5 & 57.1 & 56.4 & 54.4 \\
\hline
\end{tabular}

While the above analysis is based on observations from Sun's Fortran implementation of intervals it should be stressed that issues 2-4 are likely to occur for interval implementations based on $\mathrm{C}++$ template libraries. Specifically these will invariably replace each interval operation by a function call which performs the basic operations under directed rounding, but in so doing the presence of the special function seriously limits the possibility of loop unrolling and other advanced compiler optimizations.

With the above performance data in mind, an obvious strategy when using intervals for large scale applications would be to rewrite selective kernels removing the use of separate function calls, minimizing the need for rounding mode to be switched, and generally enhancing the ability of the compiler to optimize the code. For example a modified version of the addition kernel would look like: 


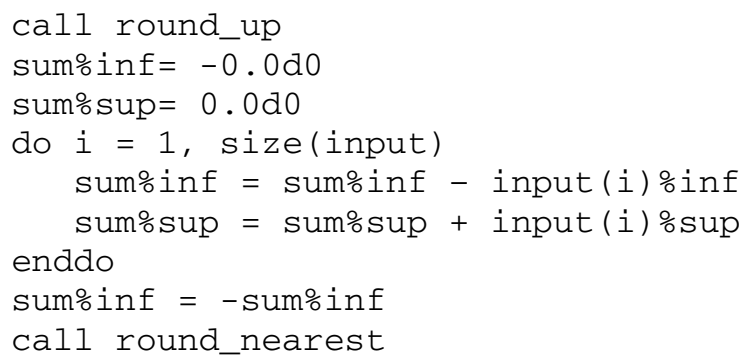

In this code the rounding mode is changed once before the loop and once at the end, and to enable only one rounding mode to be used one of the end points is negated before and after the loop (this trick is also used by the Sun compiler).

There is, however, one caveat that should be noted when attempting to hand optimizing any interval code. Specifically Sun implements an extended interval arithmetic [8] that guarantees containment for operations on infinite intervals such as $[-\infty, 0]$. Dealing with infinite intervals adds a level of complexity that for the current purpose we will ignore. (Arguing that for the vast majority of computational science applications computations on infinity are not meaningful and in anycase if required this special condition can be handled by an "if test" that in most cases will be ignored given the underlying branch prediction hardware.)

Generating hand optimized code for the product benchmark is a little bit more complex as there are at least two alternative interval product formulations [7]. The first is a direct translation of the min/max operations given in Equation 2, while the second recognizes that depending on the signs of the two infima and two suprema it is usually only necessary to perform two multiplications in order to obtain the result. An exception arises when both operands span zero, in which case four multiplications are required. This scheme requires multiple if tests so will be referred to as the branching approach. For the purpose of generating hand optimized code for the product benchmark both options have been programmed.

At this point as well as considering the performance of the hand optimized sum and product kernels it is pertinent also to consider the performance of the Sun Fortran 95 intrinsic sum and product functions, since these perform identical functions to the original kernel. Thus in Table 3 we compare timings obtained from the basic interval code, with those obtained using the intrinsic functions and hand optimized versions of these routines. These show that the performance obtained using the intrinsic sum is significantly better than that obtained by the initial benchmark code. Indeed for the smallest vector size the intrinsic function outperforms the hand optimized code by $40 \%$, but for the larger cases the hand optimized code is superior. The reason for this is that the hand optimized code has been compiled to include prefetch instructions, while the intrinsic function does not (evident from the assembly). Thus for the small benchmark where data is drawn from level 1 cache the intrinsic function shows superior performance, while for the larger benchmarks the processor stalls waiting for data to arrive in registers. For the hand optimized summation code the performance is now within a factor of four from the equivalent floating point code, and while this is larger than we might have expected, it is significantly better than our initial results.

For the product use of the intrinsic function is roughly twice as fast as the original code if the data is in cache. The hand optimized $\mathrm{min} / \mathrm{max}$ approach appears to give the 
best performance, although it is still approximately 16 times slower than the equivalent floating point code. In comparison to $\mathrm{min} / \mathrm{max}$ the branching approach is slightly slower, but this is probably not surprising given that exactly what branches are taken depends on the data values and if these are random (which they are by design in the benchmark used here) branch prediction is ineffective. It is therefore somewhat interesting to see (via disassembly of the relevant routine) that Sun appear to have implemented their interval product using a branching approach.

Table 3. Performance (nsec) comparison of initial summation and product benchmark with versions that uses Fortran intrinsic functions and versions that are hand optimised. Codes compiled using Sun Fortran 958.1 and run on a $900 \mathrm{MHz}(1.1 \mathrm{nsec})$ UltraSPARC III Cu under Solaris 5.10.

\begin{tabular}{lccccccc}
\hline & \multicolumn{3}{c}{ Summation Benchmark } & \multicolumn{4}{c}{ Product Benchmark } \\
\cline { 5 - 7 } Name & Initial & Intrinsic & Hand & Initial & Intrinsic & Min/Max & Branch \\
\hline Small & 69.1 & 4.71 & 6.7 & 119 & 66.6 & 26.8 & 37.0 \\
Medium & 90.7 & 16.3 & 6.7 & 139 & 78.0 & 27.2 & 38.5 \\
Large & 92.3 & 73.0 & 36.6 & 141 & 136.0 & 56.6 & 48.5 \\
\hline
\end{tabular}

Noting the relative difference between the two alternative product formulations it is of interest to consider the relative performance of these two schemes on other platforms, and in particular on an out-of-order processor with speculative execution. Thus the original Sun Fortran benchmark was re-written in $\mathrm{C}$ and run on an Intel Pentium 4 based system. As this processor has only a 512KB level 2 cache results are given in Table 4 for just the small and large benchmarks sizes. These show that the branching product formulation is now significantly faster than the $\mathrm{min} / \mathrm{max}$ formulation. Comparing the Pentium 4 and UltraSPARC III $\mathrm{Cu}$ we find that the small summation benchmark runs faster on the Pentium 4 by a factor that is roughly equal to the clock speed ratio (2.6 faster compared to a clock speed ratio of 2.9). For the large summation benchmark this ratio is higher reflecting the greater memory bandwidth of the Pentium compared to the UltraSPARC. For the product the branching code is approximately 3.5 times faster than the $\mathrm{min} / \mathrm{max}$ code on the UltraSPARC.

Table 4. Performance (nsec) of hand optimized $\mathrm{C}$ benchmark compiled using gcc 3.4.4 and run on a $2.6 \mathrm{GHz}(0.385 \mathrm{nsec})$ Intel Pentium 4 system under the Linux 2.6.8-2-686-smp core

\begin{tabular}{cccc}
\hline & Sum & \multicolumn{2}{c}{ Product } \\
\cline { 3 - 4 } Name & Hand & Min/Max & Branch \\
\hline Small & 2.6 & 35.2 & 7.6 \\
Large & 6.6 & 36.3 & 8.7 \\
\hline
\end{tabular}

\section{Compound Interval Operations}

The previous section compared the performance of basic floating point and interval addition and multiplication operations. In this section we consider the dot-product and AXPY (Alpha times X plus Y) operations that form part of the level 1 Basic Linear 
Algebra Subprogram (BLAS) library. As well as being slightly more complex, these operations are of interest since they form the building blocks for higher level BLAS operations, such as matrix multiplication. While the dot product and AXPY operations were explicitly coded in Fortran, dot product is also a Fortran90 intrinsic function and an interval version of AXPY is available as part of the Sun Performance Library. Thus in Table 5 we present timing results for both these operations obtained using basic Fortran code, Sun intrinsic functions or the Sun performance library, and hand optimized versions of these routines produced using a similar strategy to that outlined above for the simple benchmarks. These results again show that significant benefit can be gained from hand optimizing these routines.

Table 5. Performance (nsec) of various floating pint and interval level 1 BLAS benchmarks compiled using Sun Fortran 958.1 and run on a $900 \mathrm{MHz}(1.1 \mathrm{nsec})$ UltraSPARC III Cu under Solaris 5.10. See text for details.

\begin{tabular}{rcccccc}
\hline & \multicolumn{2}{c}{ Floating Point } & \multicolumn{2}{c}{ Sun Interval } & \multicolumn{2}{c}{ Hand Interval } \\
Name & Dot & AXPY & Dot & AXPY & Dot & AXPY \\
\hline Small & 3.4 & 6.3 & 81.4 & 250 & 18.1 & 34.1 \\
Medium & 3.3 & 6.6 & 108 & 306 & 18.4 & 85.0 \\
Large & 14.6 & 18.6 & 216 & 314 & 94.7 & 152 \\
\hline
\end{tabular}

\section{Conclusions and Discussions}

Most software support for interval computation is in the form of libraries with all interval operations being compiled to library calls; this appears to be the approach that Sun have taken in providing Fortran support for intervals. The results obtained here show that such implementations are unlikely to result in performance levels that are acceptable for them to be used in large scale scientific computations. Better performance can be obtained by effectively inlining the interval operations, minimizing changes in rounding mode, and allowing the compiler to see and optimize as much of the time consuming interval code as possible. Such an approach does come with potential drawbacks, notably the containment problems alluded to above. For the bulk of computational science applications, however, we believe that this is not a major issue compared with the need to obtain much better performance from current interval implementations. (At least from the perspective of generating a few large scale proof of concept computation science applications that show a positive benefit from the use of intervals.)

While the performance of current interval implementations is likely to be too slow for them to find widespread use in computational science, it is of interest to consider architectural changes that might alter this conclusion. To this end in Table 6 we compare the floating point and memory operation mix required for floating point and interval based dot and AXPY operations (assuming that an interval multiplication requires 8 floating point multiplications). This shows that while on both the UltraSPARC III $\mathrm{Cu}$ and Pentium 4 processors the floating point versions are load/store limited, the interval versions tend to be floating point limited. Other nontrivial interval operations seems to exhibit similar behavior leading to us to the 
general conclusion that interval arithmetic code requires a higher ratio of floatingpoint or flow-control operations to memory operations than does the corresponding floating point code. Given that the performance of most modern computational science applications tend to be load store limited yet on chip real estate is becoming increasingly cheap (as evidenced by the development of larger on chip caches plus multithreaded and multi-core chips) raises the question as to whether with relatively little effort new chips could be designed to perform interval operations much faster.

Table 6. Comparison of operation mix for floating point and interval based Dot and AXPY level 1 BLAS operations (of length $n$ ) with hardware limits

\begin{tabular}{lrccccc}
\hline & & \multicolumn{2}{c}{ Floating-Point Ops } & \multicolumn{2}{c}{ Memory Ops } & Ratio \\
& & Add & Multiply & Load & Store & FP:Mem \\
\hline Dot & Floating & $\mathrm{n}$ & $\mathrm{n}$ & $2 \mathrm{n}$ & 1 & $1: 1$ \\
& Interval & $2 \mathrm{n}$ & $8 \mathrm{n}$ & $4 \mathrm{n}$ & 2 & $5: 2$ \\
AXPY & Floating & $\mathrm{n}$ & $\mathrm{n}$ & $2 \mathrm{n}$ & $\mathrm{n}$ & $2: 3$ \\
& Interval & $2 \mathrm{n}$ & $8 \mathrm{n}$ & $4 \mathrm{n}$ & $2 \mathrm{n}$ & $5: 3$ \\
\multirow{4}{*}{ Limit } & UltraSPARC III Cu & 1 & 1 & & 1 & \\
& Pentium 4 & 1 & 1 & & 1 & $2: 1$ \\
& & & & & & \\
\hline
\end{tabular}

Acknowledgements. This work was supported in part by the Australian Research Council through Discovery Grant DP0558228.

\section{References}

1. IEEE Standard 754-1985 for Binary Floating-point Arithmetic, IEEE, (1985). Reprinted in SIGPLAN 22, 9-25.

2. R.E. Moore, "The Automatic Analysis and Control of Error in Digital Computation Based on the Use of Interval Numbers", in "Proc. of an Adv. Seminar Conducted by the Mathematics Research Center, US Army, University of Wisconsin, Madison", Ed L.B. Rall, John Wiley, (1965), pp 61-130. (Available at http://interval.louisiana.edu/ Moores_early_papers/Moore_in_Rall_V1.pdf)

3. G.W. Walster, "Interval Arithmetic: The New Floating-Point Arithmetic Paradigm", Oct 1997, Sun Microsystems white paper, available at http://www.mscs.mu.edu/ globsol/ Papers/f90-support-clean.ps

4. Interval web page http://www.cs.utep.edu/interval-comp/main.html

5. M. Lerch, G. Tischler, and J.W. von Gudenberg, filib++ - interval library specification and reference manual. Techn. Rep. 279, Lehrstuhl für Informatik II, Universität Würzburg, 2001.

6. Fortran 95 interval arithmetic programming reference, Sun Studio 10. Tech. Rep. 8190503-10, Sun Microsystems, Inc., Jan 2005.

7. J. Milthorpe, "Using Interval Analysis to Bound Numerical Errors in Scientific Computing", honours thesis, Department of Computer Science, Australian National University, October 2005.

8. G.W. Walster, Closed Interval Systems, Sun Microsystems whitepaper, 2002, available at http://www.sun.com/software/sundev/whitepapers/closed.pdf. 